\title{
The Correlation of Basal Serum FSH, Antral Follicle Count with Ovarian Response in Women with Advanced Reproductive Age
}

TANZEEM S CHOWDHURY ${ }^{1}$, SHIRIN AKHTER BEGUM ${ }^{2}$, TACHOWDHURY ${ }^{3}$

\begin{abstract}
:
Objective (s): The aim of this study was to find out the correlation between basal serum Follicle Stimulating Hormone (FSH) level, antral follicle count and number of oocytes retrieved during IVF cycle in women with advanced reproductive age.

Method: It was a cross sectional observational study which was done between January 2015 and December 2015 in Infertility Management Center, a tertiary center in Dhaka where assisted reproductive technologies are being offered. Eighty nine (89) infertile patients who were between 35 to 45 years of age and have come for IVF treatment for the first time were included in this study. The selected patients had undergone estimation of basal serum FSH by automated immuno assay analyzer and counting of the antral follicles by transvaginal sonography on day two or three. In total sixty nine (69) patients started IVF treatment according to GnRH long agonist protocol. Controlled ovarian stimulation started with $225 \mathrm{IU}$ rFSH. Follicle monitoring was done on day 5 and day 9 and the dosage was kept same or changed according to the patient's response. After day nine of stimulation, ten women were excluded as they had no mature follicle of $18 \mathrm{~mm}$ or more and cycle was cancelled. So in fifty nine (59) cases ovulation was triggered with hCG $5000 \mathrm{IU}$ on the day when at least one mature follicle measuring $18 \mathrm{~mm}$ was observed. The ovum pickup was done 32 hours after the trigger and the number of collected oocytes was counted under microscope.

Outcome measures of this study was to compare basal FSH and antral follicle count as predictors of ovarian reserve by correlating with the number of oocytes retrieved and to correlate the age of the female partner with the number of oocytes retrieved.

Results: Most couples in this study (68.33\%) have been suffering from primary infertility and majority of them had six to ten years of infertility. Higher proportion of the female partners (75\%) was between 35 to 37 years. The majority of infertile couples have male factor infertility (32\%). The second commonest cause found was tubal factor in female partner (20\%). Stepwise multiple regression analysis was done. Significant positive correlation was noticed between AFC and number of oocytes $(b=0.2413)$. There was negative correlation between the basal FSH level and the number of oocytes $(\mathrm{b}=-0.5083)$. Age of female partner had weak correlation with ovarian reserve.
\end{abstract}

Conclusion: Measurement of antral follicle number in the follicular phase is a better predictor of ovarian reserve in comparison to basal FSH and age of the women.

Key words: antral follicles, FSH, ovarian response, oocytes

Introduction:

Female age has consistently been shown to be the most important predictor of success in assisted reproductive technologies. There have been numerous reports in last twenty years showing the age-related effect on the results of IVF treatment in infertile couples.
Templeton ${ }^{1}$ in 1996 first demonstrated that the probability of live birth obtained through IVF treatment clearly decreases after the age of 35 .

Over the past two decades, a number of hormonal markers and imaging tests have been studied for their ability to predict outcome of IVF in terms of oocyte

1. Assistant Professor, OB/GYN , BIRDEM

2. Professor, OB/GYN, BSMMU

3. Chief Consultant, OBGYN, BIRDEM 
yield and occurrence of pregnancy. Even though some of these tests have become part of the routine diagnostic procedure for infertile patients who will undergo assisted reproductive techniques, there is significant debate about the superiority of one test over other to predict ovarian reserve (OR).

OR is currently defined as the number and quality of the follicles left in the ovary at any given time. ${ }^{2}$ OR can be considered normal in conditions where stimulation with the use of exogenous gonadotrophins will result in the development of at least 8-10 follicles and the retrieval of a corresponding number of healthy oocytes at follicle puncture. ${ }^{3}$

Most in vitro fertilization (IVF) clinicians determine starting doses of gonadotropin in the first cycle of IVF based principally on the patient's age and basal FSH levels ${ }^{4}$. Estimation of basal FSH is a widely available inexpensive test, which has been used for a long time in prediction of ovarian reserve. But like any other blood test, sample collection may be bothersome and there is a possibility of discrepancy between laboratories. Newer tests like estimation of anti-Mullerian hormones $(\mathrm{AMH})$ has gained a lot of interest but it is more expensive and limited to a very few centers. So recently there is a marked interest on imaging techniques, which can actually bypass the laboratory error associated with biochemical markers. The process of folliculogenesis starts months before the actual cycle. A cohort of follicles measuring 2-5 mm is known as antral follicles develop in the beginning for each cycle, which can be detected by transvaginal ultrasound that can actually predict the ovarian reserve.

The purpose of this study was to compare basal FSH and antral follicle count as predictors of ovarian reserve by correlating with the number of oocytes retrieved in women with advanced reproductive age.

\section{Materials and Methods:}

It was a cross sectional observational study which was done between January 2015 and December 2015 in Infertility Management Center, a tertiary center in Dhaka where assisted reproductive technologies are being offered. Eighty nine (89) infertile patients, who were from 35 to 45 years of age and have come for IVF treatment for the first time were included in this study. The selected patients had undergone estimation of basal serum FSH by automated immuno assay analyzer and counting of the antral follicles by transvaginal sonography $(5 \mathrm{MH})$ on day two or three of the previous natural cycle.

Inclusion criteria of this study was

- Patients with serum FSH less than $12 \mathrm{mlU} / \mathrm{ml}$.

Exclusion criteria of this study were

- Patients with serum FSH $\geq 12 \mathrm{mlU} / \mathrm{ml}$

- History of surgical removal of one ovary

- Patients not willing to take part in the study.

In total sixty-nine (69) patients started IVF treatment according to $\mathrm{GnRH}$ long agonist protocol. Controlled ovarian stimulation started with $225 \mathrm{IUrFSH}$. Follicle monitoring was done on day 5 and day 9 and the dosage was kept same or changed according to the patient's response. After day nine of stimulation, ten women were excluded as they had no mature follicle of $18 \mathrm{~mm}$ or more and their cycle was cancelled. So in fifty nine (59) cases ovulation was triggered with hCG 5000 IU on the day when at least one mature follicle measuring $18 \mathrm{~mm}$ was observed. The ovum pickup was done 32 hours after the trigger and the number of collected oocytes was counted under microscope.

The outcome measures of this study were to compare basal FSH and antral follicle count as predictors of ovarian reserve by correlating with the number of oocytes retrieved and to correlate the age of the female partner with the number of oocytes retrieved.

Pearson Correlation Coefficients and multiple regression analysis was done by SPSS to correlate the variables.

\section{Results:}

Table I shows the different characteristics of the patients. Mean age of the patients was $36.59 \pm 1.57$ years with $75 \%$ within $35-37$ years of age. Average duration of infertility was $6.33 \pm 1.70 y e a r s$. Baseline $\mathrm{FSH}$ was $8.56 \pm 3.84 \mathrm{mIU} / \mathrm{mL}$ and number of oocyte retrieved was $6.90 \pm 5.52$. Most couples in this study $(68.33 \%)$ have been suffering from primary infertility (Fig 1).The majority of infertile couples have male factor infertility (32\%). The second commonest cause found was tubal factor in female partner (20\%). There were 17 (29\%) patients in whom no cause could be identified in either of the partners and were classified as unexplained infertility (Fig 2).In this study, total number of collected oocytes was the dependent variable and the age of the female partner, serum FSH and AFC were independent variables (Table II).Pearson's correlations were carried out between different age of 
patient, $\mathrm{FSH}$ and $\mathrm{AFC}$ as independent variables with total number of oocytes retrieved as dependent variable. Significant positive correlation was noticed between AFC and number of oocytes $(r=0.218)$. (Table II). To characterize the factors with predictive values for the number of oocytes to be obtained AFC, basal $\mathrm{FSH}$ concentration and the age of patients were entered in the multiple regression analysis, in which the number of oocytes obtained was defined as a dependent variable. It was noted that AFC yielded the highest positive regression coefficient $(b=0.2413)$ (Table III)

Table-I

Patients characteristics and number of oocyte retrieved

\begin{tabular}{lc}
\hline Characteristics & Mean \pm SD \\
\hline Age (Yrs) & $36.59 \pm 1.57$ \\
Duration of infertility (Yrs) & $8.07 \pm 4.57$ \\
Basal FSH (mIU/mL) & $6.33 \pm 1.70$ \\
Basal antral follicle count $(\mathrm{N})$ & $8.56 \pm 3.84$ \\
Number of oocytes retrieved $(\mathrm{N})$ & $6.90 \pm 5.52$ \\
\hline
\end{tabular}

Table-II

Pearson Correlation Coefficients of Oocyte count with relevant parameters $(n=59)$

\begin{tabular}{lcccc}
\hline & Oocyte & Age & FSH & AFC \\
\hline Oocyte & 1 & -0.091 & -0.178 & 0.218 \\
Age & -0.091 & 1 & 0.044 & -0.116 \\
FSH & -0.178 & 0.044 & 1 & -0.156 \\
AFC & 0.218 & -0.116 & -0.156 & 1 \\
\hline
\end{tabular}

Table-III

Multiple regression analysis of parameters in predicting the number of oocytes

Standard

\begin{tabular}{lcccc} 
& Coefficients (b) & Error & $t$ Stat & $P$-value \\
\hline Oocyte & $16.7233(\mathrm{a})$ & 17.3829 & 0.9621 & 0.3403 \\
Age & -0.2699 & 0.4647 & -0.5809 & 0.5638 \\
FSH & -0.5083 & 0.4295 & 1.1834 & 0.2418 \\
AFC & 0.2413 & 0.1927 & 1.2520 & 0.2160 \\
\hline
\end{tabular}

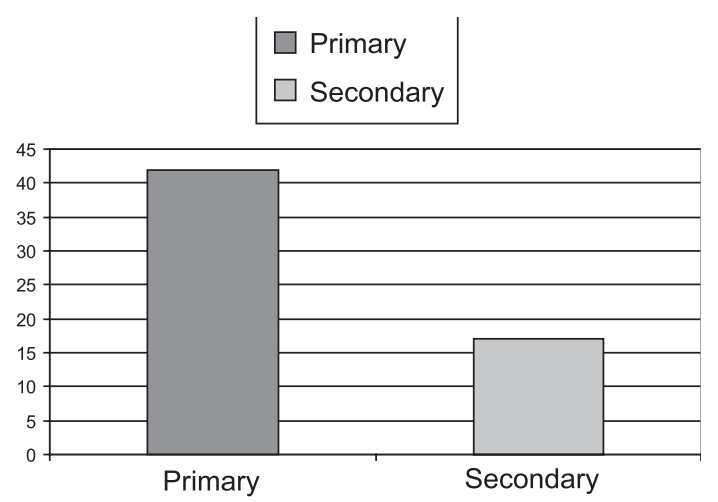

Fig.-1: Distribution of type of infertility

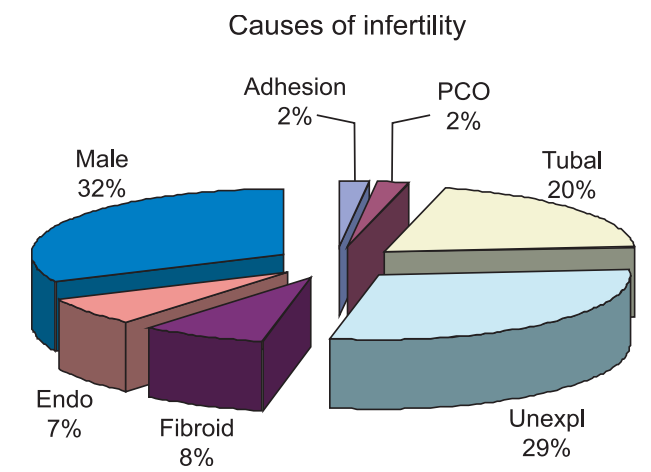

Fig.-2: Causes of infertility in the study population $(n=59)$

\section{Discussion}

Treatment of infertile couples at advanced reproductive age is a difficult job, faced by fertility specialists throughout the world. Poor ovarian response has been suggested to be associated with poor pregnancy rates and high cycle cancellation rates. ${ }^{5-8}$ So prediction of ovarian responses prior to stimulation is absolutely necessary in tailoring the dose of gonadotrophin in each individual patient. Therefore,hormonal and ultrasound parameters have been extensively evaluated by physicians for their feasibility to predict ovarian responses during ovarian stimulation.

It has been suggested that female age is also one of the important predictive parameters in ovarian response. Indeed, women's fertility is remarkably reduced along with the increase of age in both spontaneous conceptions ${ }^{9}$ and assisted reproductive methods ${ }^{1,10}$ apparently due to the decrease for the number of primordial follicles and antral follicles with size $\geq 2 \mathrm{~mm}$. In our data, there is a weak negative correlation between age of subjects with the number of oocytes retrieved $(b=-0.2699)$. 
Traditionally, early phase serum follicle stimulating hormone (FSH) levels are considered to be the most useful parameters for the prediction of ovarian reserve. ${ }^{11-13}$ Particularly, early FSH concentration is thought to be the most powerful predictive marker for assessing ovarian response as compared with that of patient age. ${ }^{14,15}$ In our study the basal FSH level had higher negative regression coefficient with the number of oocytes $(b=-0.5083)$ in comparison to female age alone.

In this study, stepwise multiple regression analysis showed that the number of antral follicles in early follicular phase had strong regression coefficient $(b=$ 0.2413 ) with number of oocytes retrieved. Studies done by Eh and Chan in 2005 has also demonstrated the significant predictive value for antral follicle count (AFC) in ovarian response and pregnancy rate of patients with in vitro fertilization (IVF) treatment, in which AFC achieved the best predictive value, followed by basal FSH, body mass index (BMI) and age of the patient. ${ }^{16}$

Many other workers around the world demonstrated that $\mathrm{AFC}$ is strongly correlated with ovarian response. They concluded that AFC is a better predictor for ovarian response than that of ovarian volume or age alone. ${ }^{17}$ Chang and colleagues found that patients with antral follicle number $\leq 3$ had a significantly higher rate of cycle cancellation and higher human menopausal gonadotropin (HMG) dosage as compared with those patients with antral follicle number 4-10 or $\geq 10 .{ }^{18}$ Similarly, Tomas and colleagues also demonstrated that the number of antral follicles correlated more strongly with the number of recovered oocytes $(r 2=0.131 ; P=0.0001)$ than age alone $(r 2=$ $-0.053 ; P=0.005)$. Fewer oocytes were recovered from patients with inactive ovaries $(5.4+/-2.5$; $\mathrm{P}=$ $0.006)$ than with normal $(7.5+/-4.5)$ or PCO-like ovaries (10.5+/-5.1). Ovarian volume was correlated with the number of follicles before stimulation $(P=$ 0.0001 ), but not with the number of oocytes. ${ }^{19}$ Most recently(2005) in Turkey, Oner and Ulug again showed that the sensitivity and specificity for prediction of ovarian response were $81 \%$ and $78 \%$ for $\mathrm{AFC}$ at an optimum cutoff value of $\leq 13.1 .^{20}$

Benaglia et al did an interesting study recently and showed that antral follicle count is the best predictor of ovarian reserve, which even in ovaries with endometriomas or with a past history of surgical excision of endometriomas. They concluded that the accuracy of AFC for predicting ovarian response is similar in unaffected ovaries, ovaries with endometriomas and ovaries with a history of surgery for endometriomas. ${ }^{21}$ These data provided additional evidence supporting that AFC is a valuable marker in ovarian response prediction.

Cancellation of cycle due to non-development of follicle in spite of optimum dose of gonadotrophins indicates poor ovarian response due to poor OR. In this regard ten cancelled patients should be included to show the relationship of FSH, AFC and age with ovarian response. But as we wanted to show the relationship of $\mathrm{FSH}, \mathrm{AFC}$ and age with number of egg retrieval, we excluded the cases where no follicle developed.

So in conclusion, this study showed that both AFC and baseline $\mathrm{FSH}$ are good predictors for the outcome of ovarian stimulation in IVF cycles. However, AFC appears to have the best predictive value. Transvaginal estimation of antral follicles is an easy and reliable method, which is a better predictor of ovarian reserve in comparison to basal $\mathrm{FSH}$, female age in women with advanced reproductive age. So this can be used routinely to predict ovarian response and guide future ovarian stimulation in a low resource set up like Bangladesh.

In this study the number of the oocytes retrieved during ovum pickup was the main outcome. The true relation between quality of oocytes and their ability to fertilize, however, remains a source of debate. Additional prospective studies with larger sample size would be necessary to further validate the conclusion. In a developing country like Bangladesh where economic resources are limited, IVF is an expensive treatment method. So this is very important to rationalize the investigations prior to initiation of treatment.

\section{Reference:}

1. Templeton A, Morris JK and Parslow W. Factors that affect outcome of in-vitro fertilization treatment. Lancet .1996; 348, 1402-1406.

2. Broekmans $\mathrm{FJ}^{1}$, Kwee J, Hendriks DJ, Mol BW, Lambalk CB. A systematic review of tests predicting ovarian reserve and IVF outcome.Hum Reprod Update. 2006;12(6):685-718.

3. Fasouliotis SJ, Simon A and Laufer. Evaluation and treatment of low responders in assisted 
reproductive technology: a challenge to meet. J Assist Reprod Genet. 2000; 17:357-373.

4. Borini A, Dal PL. Tailoring FSH and $\mathrm{LH}$ administration to individual patients. Reprod Biomed Online. 2005; 11:283-93. doi: 10.1016/ S1472-6483(10)60835-8

5. Keay SD, Liversedge NH, Mathur RS, Jenkins JM. Assisted conception following poor ovarian response to gonadotrophin stimulation. $\mathrm{Br} \mathrm{J}$ Obstet Gynaecol. 1997; 104:521-7.

6. Tarlatzis BC, Zepiridis L, Grimbizis G, Bontis J. Clinical management of low ovarian response to stimulation for IVF: a systematic review. Hum Reprod Update. 2003; 9:61-76

7. Kamble L, Gudi A, Shah A, Homburg R. Poor responders to controlled ovarian hyperstimulation for in vitro fertilization (IVF) Hum Fertil (Camb) 2011;14:230-245

8. Oudendijk JF, Yarde F, Eijkemans MJ, Broekmans FJ, Broer SL. The poor responder in IVF: is the prognosis always poor? A systematic review. Hum Reprod Update. 2012; 18:1-11.

9. Toner JP, Flood JT. Fertility after the age of 40. Obstet Gynecol Clin North Am. 1993; 20:261272.

10. Pearlstone AC, Oei ML, Wu TC. The predictive value of a single, early human chorionic gonadotropin measurement and the influence of maternal age on pregnancy outcome in an infertile population. Fertil Steril. 1992; 57:302-304.

11. Hofmann GE, Scott RT Jr, Horowitz GM, Thie J, Navot D. Evaluation of the reproductive performance of women with elevated day 10 progesterone levels during ovarian reserve screening. Fertil Steril. 1995; 63:979-983.

12. Padilla SL, Garcia JE. Effect of maternal age and number of in vitro fertilization procedures on pregnancy outcome. Fertil Steril. 1989; 52: 270-273
13. Sharara FI, Scott RT Jr, Seifer DB. The detection of diminished ovarian reserve in infertile women. Am J Obstet Gynecol. 1998; 179:804-812.

14. Cahill DJ, Prosser CJ, Wardle PG, Ford WC, Hull MG. Relative influence of serum follicle stimulating hormone, age and other factors on ovarian response to gonadotrophin stimulation. Br J Obstet Gynaecol. 1994; 101:999-1002.

15. Sharif $K$, Elgendy M, Lashen $H$, Afnan M. Age and basal follicle stimulating hormone as predictors of in vitro fertilization outcome. $\mathrm{Br} \mathrm{J}$ Obstet Gynaecol. 1998; 105:107-112

16. $\mathrm{Ng} \mathrm{EH}$, Chan CC, Tang OS, Ho PC. Antral follicle count and FSH concentration after clomiphene citrate challenge test in the prediction of ovarian response during IVF treatment. Hum Reprod. 2005; 20:1647-1654.

17. $\mathrm{Ng} \mathrm{EH}$, Tang OS, Ho PC. The significance of the number of antral follicles prior to stimulation in predicting ovarian responses in an IVF programme. Hum Reprod. 2000; 15:1937-1942.

18. Chang MY, Chiang $\mathrm{CH}$, Hsieh TT, Soong YK, $\mathrm{Hsu} \mathrm{KH}$. Use of the antral follicle count to predict the outcome of assisted reproductive technologies. Fertil Steril. 1998; 69:505-510.

19. Tomas C, Nuojua-Huttunen S, Martikainen H. Pretreatment transvaginal ultrasound examination predicts ovarian responsiveness to gonadotrophins in in-vitro fertilization. Hum Reprod. 1997; 12:220-223.

20. Oner G, Ulug P, Elmali F. Ovarian reserve markers in unexplained infertility patients treated with clomiphene citrate during intrauterine insemination. Arch Med Sci 2015; 11(6): 1250-4.

21. Benaglia L, Candotti G, Busnelli A, Paffoni A, Vercellini $P$, Somigliana E. Antral follicle count as a predictor of ovarian responsiveness in women with endometriomas or with a history of surgery for endometriomas. Fertil Steril. 2015;103(6):1544-50. 